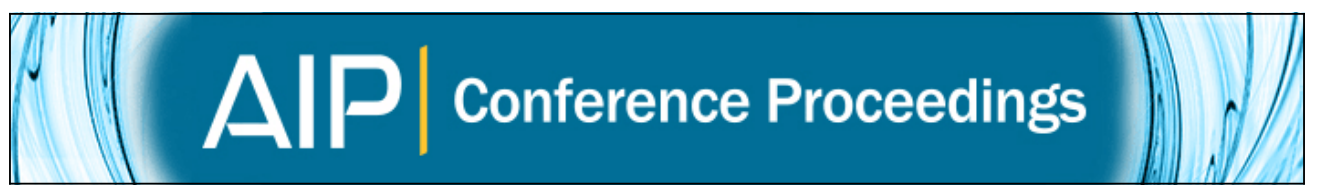

\title{
Gravitational Collapse of Prolate Brill Waves
}

Oliver Rinne

Citation: AIP Conference Proceedings 1122, 380 (2009); doi: 10.1063/1.3141331

View online: http://dx.doi.org/10.1063/1.3141331

View Table of Contents:

http://scitation.aip.org/content/aip/proceeding/aipcp/1122?ver=pdfcov

Published by the AIP Publishing

\section{Articles you may be interested in}

Preface: IX Mexican School on Gravitation and Mathematical Physics: Cosmology for the XXIst Century

AIP Conf. Proc. 1548, 1 (2013); 10.1063/1.4817030

Dyadosphere formed in gravitational collapse

AIP Conf. Proc. 1059, 72 (2008); 10.1063/1.3012287

Gravitational Wave Sources: An Overview

AIP Conf. Proc. 686, 3 (2003); 10.1063/1.1629411

Constraining post-Newtonian parameters with gravitational waves

AIP Conf. Proc. 586, 802 (2001); 10.1063/1.1419661

Brill wave initial-value problem

AIP Conf. Proc. 493, 306 (1999); 10.1063/1.1301602 


\title{
Gravitational Collapse of Prolate Brill Waves
}

\author{
Oliver Rinne \\ Department of Applied Mathematics and Theoretical Physics, Centre for Mathematical Sciences, \\ Wilberforce Road, Cambridge CB3 OWA, UK \\ King's College, Cambridge CB2 1ST, UK \\ Theoretical Astrophysics 130-33, California Institute of Technology, 1200 East California \\ Boulevard Pasadena, CA 91125, USA
}

\begin{abstract}
It has been conjectured that the gravitational collapse of sufficiently prolate vacuum axisymmetric gravitational waves (Brill waves) may violate cosmic censorship. Improving on earlier work by Garfinkle and Duncan, I present a numerical evolution of such a prolate initial data set that does form an apparent horizon. Related advances in the construction of axisymmetric constrained evolution schemes are also discussed.
\end{abstract}

Keywords: Numerical relativity, cosmic censorship, constraint equations

PACS: 04.25.D-, 04.20.Cv, 04.20.Ex, 04.25.de

\section{INTRODUCTION}

One of the major outstanding problems in classical general relativity is the validity of the cosmic censorship conjecture [1], which states that the future development of generic initial data does not contain any naked singularities. Various candidates for a violation of this conjecture have been proposed. A particularly simple example are asymptotically flat axisymmetric vacuum spacetimes containing gravitational waves, the so-called Brill waves [2]. Abrahams et al. [3] constructed numerically a sequence of increasingly prolate Brill wave initial data sets. None of these contained an apparent horizon but it appeared that the curvature could take on arbitrarily large values. Hence the authors conjectured that sufficiently prolate Brill wave initial data would evolve to form a naked singularity. Garfinkle and Duncan [4] performed a numerical evolution of one of these highly prolate initial data sets. They found indications that an apparent horizon would form eventually, but were unable to evolve long enough. In Sec. 4 I present a numerical evolution of the same initial data all the way up to the formation of an apparent horizon. It follows [5] that an event horizon exists, so that cosmic censorship is satisfied.

This extended numerical evolution was made possible by an improved understanding of constrained evolution schemes, whereby the Einstein constraint equations are solved at each time step - the approach that has been adopted in most previous work on axisymmetric gravitational collapse $[4,6]$. As I shall show in Sec. 3, these earlier schemes contained certain indefinite elliptic equations. Such equations admit potentially nonunique solutions and cause problems when attempting to solve them numerically (Sec. 2). In Sec. 3 I sketch an improved scheme that does not suffer from this problem.

For further details on this work, the reader is referred to Ref. [7]. Similar ideas have recently been applied to a fully $(3+1)$-dimensional (i.e. without symmetries) constrained evolution scheme [8]; see also I. Cordero-Carrión's talk at this conference. 


\section{2. (IN)DEFINITE ELLIPTIC EQUATIONS}

The elliptic equations encountered in the axisymmetric constrained evolution schemes discussed below in Sec. 3 are essentially of the quasi-Helmholtz type

$$
\Delta u+F(u)=f
$$

where $u \in \mathbb{R}^{2}, \Delta$ is the flat-space Laplace operator, and $F$ and $f$ are smooth functions. Suppose $u_{0}$ is a given solution and consider a small perturbation of it, $u=u_{0}+\delta u$. Taylor-expanding, $F(u) \approx F\left(u_{0}\right)+F^{\prime}\left(u_{0}\right) \delta u$. Hence for $u$ to be a solution of Eq. (1) as well, we must have

$$
\Delta \delta u+F^{\prime}\left(u_{0}\right) \delta u=0 .
$$

By the maximum principle, this only has the trivial solution $\delta u=0$ if $F^{\prime}\left(u_{0}\right) \leqslant 0$. In this case Eq. (1) is linearization stable, a necessary criterion for uniqueness of solutions. If $F^{\prime}(u) \leqslant 0$ for all $u$ then Eq. (1) is said to be definite, otherwise it is indefinite.

Apart from potentially admitting non-unique solutions, indefinite elliptic equations also lead to a loss of convergence of numerical solvers based on classical relaxation methods such as the Gauss-Seidel method (see Ref. [7] for details).

\section{AN IMPROVED CONSTRAINED EVOLUTION SCHEME}

For the case where the Killing vector is hypersurface orthogonal, the line element of an axisymmetric spacetime can be written in cylindrical polar coordinates $t, r, z, \phi$ as

$$
\mathrm{d} s^{2}=-\alpha^{2} \mathrm{~d} t^{2}+\psi^{4}\left\{\mathrm{e}^{2 r S}\left[\left(\mathrm{~d} r-\beta^{r} \mathrm{~d} t\right)^{2}+\left(\mathrm{d} z-\beta^{z} \mathrm{~d} t\right)^{2}\right]+r^{2} \mathrm{~d} \phi^{2}\right\} .
$$

Here $\alpha$ is the lapse, $\beta^{A}$ the shift $(A=r, z), \psi$ the conformal factor, and $S$ represents the dynamical degree of freedom. We have imposed quasi-isotropic gauge $\gamma_{r z}=0$ and $\gamma_{r r}=\gamma_{z z}$ on the spatial metric $\gamma$. Preservation of this gauge condition implies

$$
\beta_{+} \equiv \beta_{, r}^{z}+\beta_{, z}^{r}=2 \alpha K_{r}^{z}, \quad \beta_{-} \equiv \beta_{, r}^{r}-\beta_{, z}^{z}=-\alpha U,
$$

where $K^{z}$ and $U \equiv K^{z}{ }_{z}-K^{r}{ }_{r}$ are components of the extrinsic curvature. In addition one usually imposes maximal slicing $K_{i}^{i}=0(i=r, z, \phi)$; preservation of this condition under the time evolution implies an elliptic equation for the lapse,

$$
\begin{array}{r}
\alpha_{, r r}+\alpha_{, z z}+\left(2 \psi^{-1} \psi_{, r}+r^{-1}\right) \alpha_{, r}+2 \psi^{-1} \psi_{, z} \alpha_{, z} \\
-2 \alpha \psi^{4} \mathrm{e}^{2 r S}\left[\frac{1}{3}\left(U+\frac{1}{2} r W\right)^{2}+\frac{1}{4}(r W)^{2}+\left(K_{r}^{z}\right)^{2}\right]=0
\end{array}
$$

where we have also introduced $W \equiv\left(K^{r}{ }_{r}-K^{\phi} \phi\right) / r$. As such, Eq. (5) is definite in the sense of Sec. 2 because the factor multiplying $\alpha$ in the second line is non-negative.

In their fully constrained evolution scheme, Choptuik et al. [6] chose to eliminate the extrinsic curvature variables $K^{z}{ }_{r}$ and $U$ in favor of (first derivatives of) the shift using Eq. (4), thus obtaining an equation of the form

$$
\alpha_{, r r}+\alpha_{z z}+\ldots-\psi^{4} \mathrm{e}^{2 r S} \alpha^{-1}\left[\frac{2}{3} \beta_{-}^{2}+\frac{1}{2} \beta_{+}^{2}\right]+\ldots=0 .
$$


Because of the negative power of $\alpha$, this equation is indefinite in the sense of Sec. 2. Instead of using Eq. (4) to eliminate $K^{z}{ }_{r}$ and $U$, I propose to introduce a new vector $\eta^{A}$ obeying

$$
\eta_{+} \equiv \eta^{z}{ }_{r}+\eta_{, z}^{r}=2 K_{r}^{z}, \quad \eta_{-} \equiv \eta_{, r}^{r}-\eta_{, z}^{z}=-U .
$$

Since there is no $\alpha$ dependence in these equations, substituting them in Eq. (5) will not destroy the definiteness of that equation. The momentum constraints give rise to a pair of elliptic equations for $\eta^{A}$, and then the isotropic gauge conditions (4) are solved for $\beta^{A}$.

A different problem is caused by the Hamiltonian constraint equation, which reads

$$
\begin{array}{r}
\psi_{, r r}+\psi_{z z}+r^{-1} \psi_{, r}+\frac{1}{4}\left[(r S)_{, r r}+(r S)_{, z z}\right] \\
+\frac{1}{4} \psi^{5} \mathrm{e}^{2 r S}\left[\frac{1}{3}\left(U+\frac{1}{2} r W\right)^{2}+\frac{1}{4}(r W)^{2}+\left(K^{z}\right)^{2}\right]=0 .
\end{array}
$$

This equation is of indefinite type. It can be cured by introducing conformally rescaled extrinsic curvature variables (cf. Ref. [9]):

$$
\left\{\tilde{K^{z}}, \tilde{U}, \tilde{W}\right\} \equiv \psi^{6}\left\{K^{z}, U, W\right\} .
$$

When Eq. (8) is written in terms of $\tilde{K}_{r}^{z}, \tilde{U}$ and $\tilde{W}$, the power $\psi^{5}$ is changed into $\psi^{-7}$, and the equation becomes definite. Instead of the original extrinsic curvature variables, the conformally rescaled ones are evolved. The particular value of the exponent of $\psi$ in Eq. (9) has been chosen so that the momentum constraints decouple from the remaining elliptic equations, which then take on a hierarchical structure [7].

\section{NUMERICAL EVOLUTION OF PROLATE BRILL WAVES}

The new constrained evolution scheme outlined above has been implemented using second-order accurate finite differences, a multigrid elliptic solver, Crank-Nicholson time integration, combined with (fixed) mesh refinement (see Ref. [7] for details).

The prolate Brill wave evolution shown here has initial data at a moment of time symmetry given by

$$
S=\operatorname{ar} \exp \left(-r^{2} / \sigma_{r}^{2}-z^{2} / \sigma_{z}^{2}\right)
$$

with $a=325, \sigma_{r}=0.128$, and $\sigma_{z}=1.6$, corresponding to an ADM mass of $M_{\mathrm{ADM}} \approx 2$. Figure 1 shows the time evolution of the lapse function and the Kretschmann scalar $I \equiv R^{a b c d} R_{a b c d} / 16$ evaluated in the origin, indicating that a singularity is approached but that the curvature actually decreases. Figure 2 shows the numerically computed ADM mass as well as the mass $M_{\mathrm{AH}}$ of the apparent horizon from when it forms at $t=3.9$. The ratio $M_{\mathrm{AH}} / M_{\mathrm{ADM}} \approx 0.96$. Also shown in Fig. 2 is the coordinate location of the apparent horizon when it forms at $t=3.9$ and when the simulation was terminated at $t=4.5$.

\section{ACKNOWLEDGMENTS}

I wish to thank Sergio Dain, David Garfinkle, José Luis Jaramillo, John Stewart, and Darragh Walsh for helpful discussions on this work. A travel grant from the Gravitational Waves Section of the European Network of Theoretical Astroparticle Physics / 

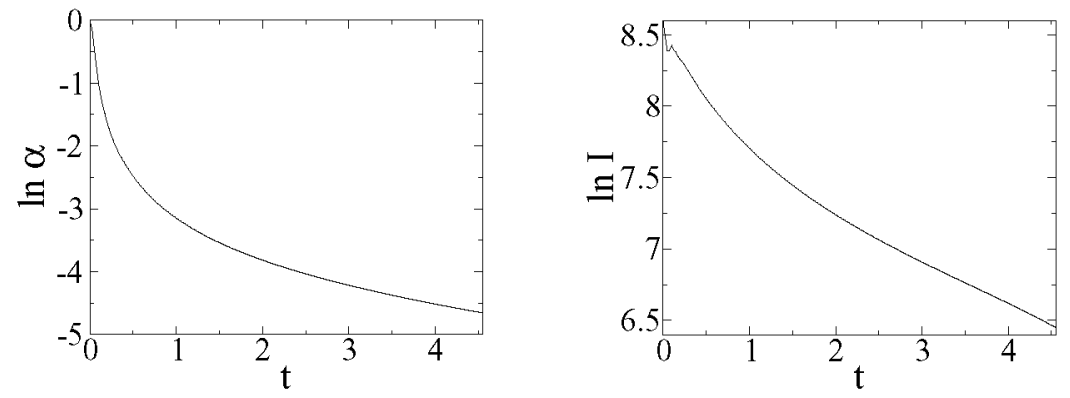

FIGURE 1. Lapse function $\alpha$ and Kretschmann scalar $I$ in the origin.
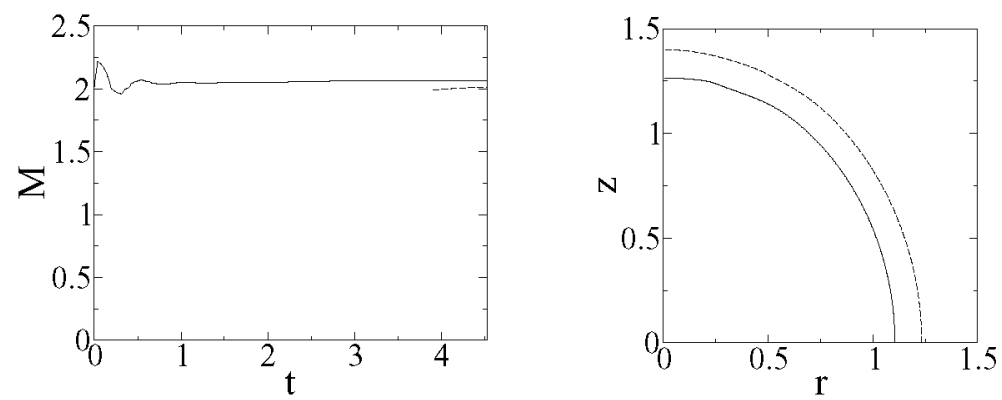

FIGURE 2. Left: ADM mass (solid) and apparent horizon mass (dashed). Right: Coordinate location of the apparent horizon when it forms at $t=3.9$ (solid), and at $t=4.5$ (dashed).

ILIAS to attend this conference is gratefully acknowledged, as is a Research Fellowship at King's College, Cambridge. Earlier parts of this work were supported by grants to Caltech from the Sherman Fairchild Foundation, NSF grant PHY-0601459, and NASA grant NNG05GG52G.

\section{REFERENCES}

1. R. Penrose, Riv. Nuovo Cimento 1, 252-276 (1969).

2. D. R. Brill, Ann. Phys. 7, 466-483 (1959).

3. A. M. Abrahams, K. R. Heiderich, S. L. Shapiro, and S. A. Teukolsky, Phys. Rev. D 46, 2452-2463 (1992).

4. D. Garfinkle and G. C. Duncan, Phys. Rev. D 63, 044011 (2001).

5. S. W. Hawking and G. F. R. Ellis, The large scale structure of space-time, Cambridge University Press, 1973.

6. M. W. Choptuik, E. W. Hirschmann, S. L. Liebling, and F. Pretorius, Class. Quantum Grav. 20, 1857 1878 (2003).

7. O. Rinne, Class. Quantum Grav. 25, 135009 (2008).

8. I. Cordero-Carrión, P. Cerdá-Durán, H. Dimmelmeier, J. L. Jaramillo, J. Novak, and E. Gourgoulhon (2008), arxiv: 0809.2325.

9. J. W. York, Jr., in Sources of gravitational radiation, edited by L. L. Smarr, Cambridge University Press, 1979, pp. 83-126. 